\title{
Diversidade de Culicidae durante os períodos crepusculares em bioma de Floresta Atlântica e paridade de Anopheles cruzii (Diptera: Culicidae) ${ }^{1}$
}

\author{
Ana C. D. Bona ${ }^{2} \&$ Mário A. Navarro-Silva ${ }^{2}$ \\ ${ }^{1}$ Contribuição número 1773 do Departamento de Zoologia, Universidade Federal do Paraná. \\ 2 Departamento de Zoologia, Universidade Federal do Paraná. Caixa Postal 19020, 81531-980 Curitiba, Paraná, Brasil. \\ E-mail: mnavarro@ufpr.br; ana.dalla@ig.com.br
}

\begin{abstract}
Diversity of Culicidae during the crepuscular periods in Atlantic Forest biome and parity of Anopheles cruzii (Diptera: Culicidae). The aim of this investigation was to know the diversity and abundance of Culicidae associating with the climatic factors and obtaining the parity of Anopheles cruzii Dyar \& Knab, 1908. The study was developed in a dense rain forest in Atlantic Forest, located in the State of the Paraná, named of Palmito State Park. The captures were performed two days in a month, during December 2006 to March 2007, occurring in the matutine and vespertine crepuscular periods. The captures were executed before twilight until after twilight. Twenty five species that belong to Culicidae were identified, the tree most abundant species were Anopheles cruzii (65.2\%), Culex sachettae Sirivanakarn \& Jacob, 1982 (11.2\%) e Anopheles bellator Dyar \& Knab, 1906 (8.5\%). According to variance analysis, significant difference in frequency, was detected between crepuscular periods, for the following species: Aedes scapularis (Rondone, 1848) $(\mathrm{p}=0.03651)$, Coquillettidia chrysonotum (Peryassu, 1922) ( $\mathrm{p}=0.00795)$, Mansonia fonsecai (Pinto, 1932) ( $\mathrm{p}=0.00804)$, e Runchomyia theobaldi Lane \& Cerqueira, 1934 $(p=0.01996)$. The tree most captured species didn't show a significant correlation to the climatic factors. The rate parity of Anopheles cruzii was $48 \%$. Didn't have correlation between the abundance with the rate parity. The twilight influences the behavior of Culicidae species. The similarity comparison between matutine and vespertine crepuscular periods revealed an elevated resemblance in the specific composition. $A$. cruzii was dominant specie in the crepuscular periods. The diversity in the crepuscular periods was considered decrease.
\end{abstract}

KEY WORDS. Culex sacchettae; parity.

RESUMO. O objetivo da investigação foi analisar a variação da diversidade e abundância das espécies de Culicidae e sua relação com algumas variáveis ambientais, bem como estimar a taxa de paridade de Anopheles cruzii Dyar 8 Knab, 1908. O estudo foi desenvolvido em Floresta Ombrófila Densa da Mata Atlântica, localizada no Estado do Paraná, denominada de Floresta Estadual do Palmito. As capturas foram executadas quinzenalmente, de dezembro 2006 a março 2007, com a técnica de "aspiração menor", nos crepúsculos vespertino e matutino, iniciando antes dos crepúsculos e finalizando após os crepúsculos. Foram detectadas 25 espécies, sendo as mais abundantes, Anopheles cruzii $(65,2 \%)$, Culex sachettae Sirivanakarn 8 Jacob, 1982 (11,2\%) e Anopheles bellator Dyar \& Knab, 1906 $(8,5 \%)$. De acordo com análise de variância, ocorreu diferença significativa na frequiência entre os períodos crepusculares para seguintes espécies: Aedes scapularis (Rondone, 1848) $(\mathrm{p}=0,03651)$, Coquillettidia chrysonotum (Peryassu, 1922) ( $\mathrm{p}=0,00795)$, Mansonia fonsecai (Pinto, 1932) ( $\mathrm{p}=0,00804)$, e Runchomyia theobaldi Lane \& Cerqueira, 1934 ( $\mathrm{p}$ $=0,01996)$. Não houve correlação significativa com a abundância das principais espécies capturadas e as médias dos fatores abióticos avaliados. A taxa de paridade de Anopheles cruzii atingiu média percentual de $48 \%$, não existindo correlação entre a abundância e a taxa de paridade. $O$ crepúsculo exerce influência no comportamento apetente das espécies de Culicidae. A comparação de similaridade entre os crepúsculos apontou para elevada semelhança da composição específica. Anopheles cruzii atingiu dominância nos períodos vespertino e matutino, sendo a diversidade de forma geral reduzida nestes períodos.

PALAVRAS-CHAVE. Culex sacchettae; paridade.

O bioma Floresta Atlântica é considerado de elevada diversidade, o qual potencialmente apresenta multiplicidade de opções de nichos para o desenvolvimento de imaturos de Culicidae, assim como da fauna de vertebrados que podem ser utilizados como hospedeiros para exercício de hematofagia. Por outro lado, como estas áreas estão sujeitas às alterações ambientais provocadas pelo avanço das atividades antrópicas em seu meio, podem estar operando mudanças na dinâmica 
das populações de vetores. Repercutindo negativamente na população humana do entorno ou que desenvolve atividade na própria área, devido ao fato dos mosquitos serem vetores de agentes etiológicos que causam doenças aos homens e animais.

Investigações que priorizam o conhecimento da diversidade de Culicidae podem revelar novas espécies, assim como hábitos desconhecidos, podem ser elucidados (Hutchins et al. 2005).

Duas espécies encontradas em áreas de Mata Atlântica da planície litorânea do Paraná apresentam destacada importância em saúde pública. Aedes scapularis (Rondone, 1848), envolvida na transmissão de arbovírus, como encefalite eqüina venezuelana, febre amarela, vetor secundário da filariose bancroftiana, vírus do Rocio no Sudeste do Brasil, e recentemente investigado como vetor da Dilofilaria imitis (SAN'T ANA \& Lozover 2001). A outra espécie é Anopheles cruzii Dyar \& Knab, 1908 importante epidemiologicamente no Sudeste e Sul do Brasil (CHAHAD-EHLes et al. 2007), envolvida com a transmissão do agente etiológico da malária humana e simiana no Sudeste do Brasil (CARvalHo-PinTo \& LOUREnÇO De Oliveira 2004). Estudos realizados no ecossistema de Mata Atlântica demonstram alta freqüência de $A$. cruzii em ambientes com cobertura vegetal primitiva (ForatTini et al. 1996), onde é encontrado grande diversidade de bromeliácea.

Na metade do século passado populações de $A$. cruzii mantiveram na litoral do Paraná, endemia de malária, na época várias medidas de controle foram efetivadas, como por exemplo, a destruição de bromélias, em 1964 o controle da endemia foi atingido (RACHOU 1958). Aproximadamente 30 anos após o último estudo que levou em consideração aspectos fisiológicos de $A$. cruzii por Luz et al. (1979), faz-se necessário verificar a paridade desta espécie, já que esta reflete a intensidade de contato com o hospedeiro vertebrado (Hовск et al. 2003).

As fêmeas de Culicidae necessitam da digestão de proteínas presentes no sangue para iniciar a vitelogênese, mudanças de intensidade da luz, determinam alterações comportamentais na busca por hospedeiro para realizar a hematofagia. Espécies dos gêneros Anopheles Meigen, 1818 e Culex Linnaeus, 1758 possuem hábitos crepusculares. De maneira geral, os culicídeos tem hábitos diurnos como por exemplo espécies de Aedes Meigen, 1818, Psorophora Robineau-desvoidy, 1827 e Sabethini, finalizando sua atividade com o ocaso do sol (ForatTini 2002).

Ecótopos naturais, ou com histórico de utilização intensiva e posterior conservação, como a área sob análise, devem ser acompanhados de avaliações do impacto das modificações ambientais sobre a diversidade e hábitos dos vetores biológicos. O objetivo do estudo foi avaliar a diversidade da fauna de Culicidae, durante os períodos crepusculares, buscando associar a variação de abundância das espécies com os fatores climáticos, bem como obter parâmetros populacionais relacionados à paridade de $A$. cruzii, no litoral do Estado do Paraná.

\section{MATERIAL E MÉTODOS}

A área de estudo localizada na Unidade de Conservação de Uso Sustentável com cerca de 530 ha de vegetação nativa
(Floresta Estadual do Palmito - FEP), encontra-se no município de Paranaguá, no Litoral do Estado do Paraná, entre 25³5'S e $48^{\circ} 32^{\prime}$ W. Localiza-se no quilômetro 4, à margem da Rodovia PR-407, distando $90 \mathrm{~km}$ da cidade de Curitiba.

O clima é caracterizado como Cfa - clima subtropical; temperatura média no mês mais frio inferior a $18^{\circ} \mathrm{C}$ (mesotérmico) e temperatura média no mês mais quente acima de $22^{\circ} \mathrm{C}$, com verões quentes e com tendência a concentração de chuvas neste período, geadas pouco, contudo sem estação seca definida, de acordo com a classificação de Köeppen (IAPAR 2000).

A precipitação média anual é de $1950 \mathrm{~mm}$, sendo Janeiro e Fevereiro meses chuvosos. A média anual da umidade relativa do ar está em torno de 85\% (Boeger \& Wisniewski 2003). Este ambiente é propício a afloramentos de água, o que favorece o desenvolvimento de inúmeras plantas.

A área possui formações pioneiras com influência marinha (restinga), flúvio-marinha (manguezal) e algumas áreas antropizadas (edificações) . A vegetação original é classificada como Floresta Ombrófila Densa de Terras Baixas e caracterizase por ser pouco exuberante, com árvores de porte médio (BoEgER \& Winiewski 2002), onde epífitas e lianas são extremamente profusas e diversificadas (RoDERJAN et al. 2002).

A vegetação da reserva tem sido modificada pela ação antrópica nos últimos 100 anos, com práticas agrícolas básicas, como o cultivo de abacaxi e mandioca, baseando-se em queimadas (Simões \& Marques 2007).

As coletas foram realizadas em íntima associação com a mata nativa que circunda a área antropizada. As capturas foram conduzidas quinzenalmente, na estação do verão 2006/2007, durante os períodos vespertinos e matutinos, totalizando 40 horas de coleta. As capturas foram iniciadas uma hora antes do crepúsculo e finalizadas uma hora após o crepúsculo. Os períodos de captura foram divididos em seis partes: Pré-crepúsculo vespertino (uma hora de captura), horário que antecede o crepúsculo; Crepúsculo vespertino (30 minutos de captura), crepúsculo propriamente dito; Pós-crepúsculo vespertino (uma hora de captura), horário que sucede o crepúsculo; Pré-crepúsculo matutino (1 hora de captura); Crepúsculo matutino (30 minutos de captura); Pós-crepúsculo matutino (uma hora de captura).

As capturas foram levadas a efeito com dois operadores, sendo que um manejava o aspirador manual e o outro servia como atrativo aos culicídeos. Esta técnica chamada de "aspiração menor" (ForatTini 2002) permite a captura do mosquito em pleno vôo, ou então ao pousarem antes do início do repasto sanguíneo propriamente dito. Os aspiradores eram movidos à bateria, e possuíam potes coletores, que eram substituídos com freqüência, para evitar a perda de caracteres diagnósticos específicos dos exemplares capturados. Os culicídeos dos potes eram transferidos para gaiolas, de acordo com os períodos de captura, e transportados vivos ao Laboratório de Entomologia Médica e Veterinária, Universidade Federal do Paraná.

No laboratório todos culicídeos eram acondicionados sob temperatura e umidade controladas, com alimentação açuca-

Revista Brasileira de Zoologia 25 (1): 40-48, March, 2008 
rada (mel 10\%). Eram escolhidas aleatoriamente 15 fêmeas de Anopheles cruzii por gaiola, totalizando 45 fêmeas dissecadas por período crepuscular. Quando o número de A. cruzii capturados por gaiola era inferior a 15 , todos os exemplares vivos foram dissecados. A dissecção foi levada a termo no máximo em 24 horas após a execução das capturas, de acordo com a técnica de Detinova (CHARLwood et al. 1980), observando as terminações traqueolares e procedendo a separação das fêmeas em paridas e não paridas.

Segundo Detinova (1962) são chamadas de paridas aquelas fêmeas que já desenvolveram seus ovos pelo menos uma vez, e podem ser distinguidas das fêmeas nulíparas ou não paridas (que nunca ovipositaram) através de observações das traquéolas ovariolares. Os ovários dos mosquitos possuem rico suprimento de oxigênio, fornecido por uma rede de traquéolas. Em fêmeas nos estádios I e II de Christophers e Mer, as finas terminações traqueolares apresentam-se enoveladas. Com o desenvolvimento dos ovos além do estádio II, começa haver o desenovelamento das traquéolas, formando uma rede dispersa, sendo esta mudança irreversível. Assim o enovelamento terminal das traquéolas é uma indicação que a fêmeas é nulípara (Charlwood et al. 1980).

Os restantes dos mosquitos eram sacrificados, montados e etiquetados. A identificação dos espécimes capturados foi realizada com auxílio de chaves dicotômicas (CorRêA \& RAMALHO 1956, Lane 1953a,b, Forattini 2002, Consoli \& Lourenço de Oliveira 1994, Lane \& Whitman 1951) descrições (Forattini 2002, BARBosa et al. 2005) e por comparação com exemplares já identificados. Na identificação dos machos, foram montadas lâminas das genitálias masculinas seguindo o protocolo de montagem do laboratório de Biosistemática de Culicidae, da Faculdade de Saúde Pública, Universidade de São Paulo.

Após a confirmação das espécies de Culicidae, os mosquitos foram depositados na Coleção de Entomologia Padre Jesus Santiago Moure, Departamento de Zoologia, Universidade Federal do Paraná (DZUP).

Para o cálculo do ritmo de atividade hematófaga das fêmeas durante os períodos crepusculares, foi calculada a média de Williams $\left(X_{w}\right)$ para cada espécie, segundo Foratrini (2002). A média de Williams designa à medida correspondente a variação da média geométrica dos resultados obtidos na isca humana: $\log \left(\mathrm{X}_{\mathrm{w}}+1\right)=\Sigma \log (\mathrm{n}+1) / \mathrm{N}$, onde: $\mathrm{n}=$ número de espécimes obtido em cada coleta e $\mathrm{N}$ = número de coletas.

Para comparar a composição específica dos períodos crepusculares vespertino e matutino foi utilizado a análise de similaridade. Foi calculado o índice de similaridade qualitativa de Sorensen (CCs), baseado na presença e ausência das espécies, para analisar a similaridade específica nos períodos vespertino e matutino (Felfili et al. 1993): CCs $=2 \mathrm{c} / \mathrm{a}+\mathrm{b}$, onde: $\mathrm{a}, \mathrm{b}$ e $c$ = número de espécies no período crepuscular vespertino, crepuscular matutino e comuns aos dois períodos, respectivamente.

Para o cálculo da similaridade quantitativa foi utilizado o Índice de Morisita-Horn (CMH) foi utilizado para estabelecer o grau de semelhança entre os dois períodos de coleta, baseando-se na composição da comunidade e abundância das espécies (Volpato \& ANjos 2001): CMH = $2 \Sigma$ (ani.bni)/(da+db). (aN.bN), onde: $\mathrm{da}=\Sigma \mathrm{ani}^{2} / \mathrm{aN}^{2}, \mathrm{db}=\Sigma \mathrm{bni}^{2} / \mathrm{bN}^{2}$, ani = número de indivíduos de cada espécie no período crepuscular vespetino, bni = número de indivíduos de cada espécie no período crepuscular matutino, $\mathrm{aN}$ = número total de indivíduos no período crepuscular vespertino e $\mathrm{bN}=$ número total de indivíduos no período crepuscular matutino.

O índice de dominância de Berger-Parcker (1970) (D) foi usado para comparação entre a prevalência de determinada espécie capturada em relação aos dois períodos crepusculares vespertino e matutino: $\mathrm{D}=$ Nmáx/Ntotal x 100, onde: Nmáx = número de mosquitos da espécie mais abundante e Ntotal = número de indivíduos encontrados.

Para estimar a riqueza dos culicídeos foi utilizado o índice de diversidade de Margalef. Medida utilizada para estimar a biodiversidade de uma comunidade com base na distribuição numérica dos indivíduos das diferentes espécies (SERVICE 1993): $\mathrm{Dm}=(\mathrm{S}-1) / \mathrm{ln} \mathrm{N}$, onde $\mathrm{S}=$ número de espécies e $\mathrm{N}$ = número de indivíduos encontrados.

Para as análises dos fatores abióticos, valores de temperaturas média $\left({ }^{\circ} \mathrm{C}\right)$, máxima $\left({ }^{\circ} \mathrm{C}\right)$ e mínima $\left({ }^{\circ} \mathrm{C}\right)$, precipitação pluviométrica ( $\mathrm{mm}$ ) e umidade relativa do ar (\%), foram obtidas planilhas através do Sistema Meteorológico do Paraná (Simepar), a partir da base 25134848 em Antonina, Paraná, estação oficial mais próxima da área das capturas. Com estes dados foram calculadas médias retrospectivas para 5, 15 e 20 dias antes da execução das oito coletas e entre as datas da coletas realizadas.

Para análise de possíveis diferenças entre a abundância das três espécies mais coletadas, dentro e entre os períodos crepusculares. Assim como, para verificar a diferença entre a abundância das espécies capturadas nos dois períodos. Foi utilizando o teste de Levene, Análise de variância e Teste Tukey HSD, com nível de significância 5\%.

O coeficiente de correlação de Pearson foi utilizado para avaliar a relação entre as variáveis ambientais e a atividade das três espécies mais freqüentes. Assim como, para inferir a estrutura dos parâmetros populacionais (Abundância X Paridade) de Anopheles cruzii, e a taxa de paridade desta espécie em relação aos fatores abióticos.

\section{RESULTADOS}

Durante o período de dezembro de 2006 a março de 2007, em ambos os crepúsculos foram coletados 3479 culicídeos, distribuídos em 25 espécies pertencentes as tribos Aedini, Culicini, Mansoniini e Sabethini. Os mais abundantes foram Anophelles cruzii $(65,19 \%)$, Culex sacchettae Sirivanakarn. \& Jakob, 1981 (11,15\%) e Anopheles bellator Dyar \& Knab, 1906 (8,51\%) com ocorrência nos dois períodos analisados (Tab. I). As espécies menos abundantes foram: Aedes scapularis, Culex sachettae Sirivanakarn \& Jacob, 1982, Mansonia fonsecai (Pinto, 1932) e Wyomyia incaudata Root, 1928 (Tab. II). 
Tabela I. Espécies de Culicidae capturados, nos crepúsculos matutino e vespertino no período de dezembro de 2006 a março de 2007, na Floresta Estadual do Palmito, Paranaguá, Paraná.

\begin{tabular}{|c|c|c|}
\hline Espécies & $\mathrm{N}$ & $\%$ \\
\hline \multicolumn{3}{|l|}{ Anophelinae } \\
\hline Anopheles cruzii Dyar \& Knab, 1908 & 2268,00 & 65,19 \\
\hline Anopheles bellator Dyar \& Knab, 1906 & 296,00 & 8,51 \\
\hline Anopheles mediopunctatus Lutz, 1903) & 27,00 & 0,78 \\
\hline \multicolumn{3}{|l|}{ Culicinae: Aedini } \\
\hline Aedes scapularis (Rondani, 1848) & 142,00 & 4,08 \\
\hline Aedes serratus (Theobald, 1901) & 22,00 & 0,63 \\
\hline Psorophora ferox (Von Humboldt,1819) & 14,00 & 0,40 \\
\hline Aedes hortator (Dyar \& Knab, 1907) & 8,00 & 0,23 \\
\hline Aedes albopictus (Skuse, 1894) & 4,00 & 0,11 \\
\hline Aedes fluviatilis (Lutz, 1904) & 1,00 & 0,03 \\
\hline Psorophora albipes (Theobald, 1907) & 1,00 & 0,03 \\
\hline \multicolumn{3}{|l|}{ Culicinae: Culicini } \\
\hline Culex sacchettae Sirivanakarn \& Jacob, 1982 & 388,00 & 11,15 \\
\hline Culex imitator Theobald, 1903 & 51,00 & 1,46 \\
\hline Culex nigripalpus (Theobald,1901) & 3,00 & 0,09 \\
\hline Culex grupo coronator & 3,00 & 0,09 \\
\hline Culex ribeirensis Forattini \& Sallum, 1985 & 3,00 & 0,09 \\
\hline Culex quinquefasciatus (Say, 1823) & 1,00 & 0,03 \\
\hline \multicolumn{3}{|l|}{ Culicinae: Mansoniini } \\
\hline Mansonia fonsecai (Pinto, 1932) & 61,00 & 1,75 \\
\hline Coquillettidia chrysonotum (Peryassu,1922) & 17,00 & 0,49 \\
\hline \multicolumn{3}{|l|}{ Culicinae: Sabethini } \\
\hline Wyeomyia incaudata Root, 1928 & 132,00 & 3,79 \\
\hline Runchomyia theobaldi Lane \& Cerqueira, 1934 & 20,00 & 0,57 \\
\hline Runchomyia reversa Lane \& Cerqueira, 1942 & 10,00 & 0,29 \\
\hline Wyeomyia lopesi (Correa \& Ramalho, 1956) & 3,00 & 0,09 \\
\hline Wyeomyia abebela Dyar \& Knab, 1908 & 2,00 & 0,06 \\
\hline Wyeomyia davisi (Lane \& Cerqueira, 1942) & 1,00 & 0,03 \\
\hline Wyeomyia galvaoi (Correa \& Ramalho, 1956) & 1,00 & 0,03 \\
\hline Total & 3479,00 & 100,00 \\
\hline
\end{tabular}

No período crepuscular vespertino o número de mosquitos atingiu aproximadamente 100 indivíduos por hora, sendo capturadas 20 espécies, destas Psoropohora albipes (Theobald, 1907), Culex quinquefasciatus (Say, 1823) e Culex grupo Coronator ocorreram exclusivamente neste período. No crepúsculo matutino, o número de mosquito por hora chegou a 75 indivíduos, porém com número superior de espécies, 22 sendo cinco com ocorrência exclusiva para este crepúsculo Culex ribeirensis Forattini \& Sallum, 1985, Wyeomyia abebela Dyar \& Knab, 1908, Wyeomyia davisi (Lane \& Cerqueira, 1942), Aedes fluviatilis (Lutz, 1904) e Wyeomyia galvaoi (Correa \& Ramalho, 1956). De forma geral, para ambos os períodos de captura, número expressivo de espécies ocorreram em freqüência reduzida, com percentual relativo inferior a 5\%. Sendo Anopheles cruzii a espécie capturada em elevada freqüência em ambos os períodos crepusculares (Tab. II).

Foram capturados 60 e 54 Anopheles cruzii/hora; 12,0 e 7,3 Culex sacchettae/hora; 10,4 e 4,3 Anopheles bellator/hora durante o período crepuscular vespertino e matutino respectivamente (Tab. II).

Diferença significativa de abundância foi detectada entre os períodos crepusculares vespertino e matutino para as seguintes espécies: Aedes scapularis ( $\mathrm{p}=0,03651)$, Coquillettidia chrysonotum (Peryassu, 1922) ( $\mathrm{p}=0,00795)$, Mansonia fonsecai ( $\mathrm{p}=0,00804)$, e Runchomyia theobaldi Lane \& Cerqueira, 1934 ( $\mathrm{p}=0,01996)$ (Fig. 1).

Analisando os períodos que antecedem e sucedem os crepúsculos vespertino e matutino, e as três espécies encontradas em elevada densidade, observamos o mesmo padrão, ou seja, um aumento gradativo na abundância de $A$. cruzii, $A$. bellator e Culex sacchettae do crepúsculo vespertino para o pós-crepúsculo vespertino e um decréscimo no crepúsculo matutino para o pré-crepúsculo matutino. Para $A$. cruzii existe diferença entre o pré-crepúsculo vespertino e matutino ( $\mathrm{p}=0,03513)$; pós-crepúsculo vespertino e matutino $(\mathrm{p}=0,01233)$; pré e pós-crepúsculo vespertino ( $\mathrm{p}=0,02757$ ); pré e pós-crepúsculo matutino $(\mathrm{p}=0,01592)$. Anopheles bellator apresentou diferença significativa entre o pré e pós-crepúsculo vespertino ( $\mathrm{p}=0,01126)$; pós-crepúsculo vespertino e matutino ( $\mathrm{p}=0,00600)$. Culex sacchettae tem diferença significativa entre o pré-crepúsculo vespertino e matutino ( $\mathrm{p}=0,00235)$; pós-crepúsculo vespertino e matutino ( $\mathrm{p}=0,00066)$; pré e pós-crepúsculo vespertino ( $p=0,00017)$. Não foi detectada diferença significativa na freqüência entre o crepúsculo vespertino e matutino para as espécies acima mencionadas (Figs 2 e 3 ).

$\mathrm{O}$ índice de similaridade de Sorensen foi de 0,81 e o índice de Morisita-Horn atingiu 0,98 entre os períodos crepusculares, vespertino e matutino. Como regra geral para os dois índices, uma similaridade de 0,5 é considerada alta. E, quanto mais próximo de 1, maior a similaridade (Felfili et al. 1993). Os índices de dominância de Berger-Packer, foram 0,60 e 0,72 para o período crepuscular vespertino e matutino respectivamente. Para o período crepuscular vespertino o índice de diversidade de Margalef foi de 2,50 e para o período crepuscular matutino foi de 2,87.

As variáveis abióticas analisadas, entre os períodos de execução das capturas apresentaram as seguintes oscilações entre valores mínimos e máximos: temperatura média entre 24,1 a $26,4^{\circ} \mathrm{C}$, a temperatura máxima de 29,1 a $34,6^{\circ} \mathrm{C}$, a temperatura mínima 20,4 a $21,6^{\circ} \mathrm{C}$, e a média da umidade relativa do ar entre 84,7 a 92,7\%. A precipitação pluviométrica acumulada entre duas coletas sucessivas de $5,4 \mathrm{~mm}$ até $308,8 \mathrm{~mm}$. Os dados ambientais no período de captura apresentaram reduzida variação, ou seja, com temperaturas e precipitações elevadas.

O litoral do Paraná corresponde à faixa de terreno situada até $500 \mathrm{~m}$ de altitude, normalmente as condições do macro- 


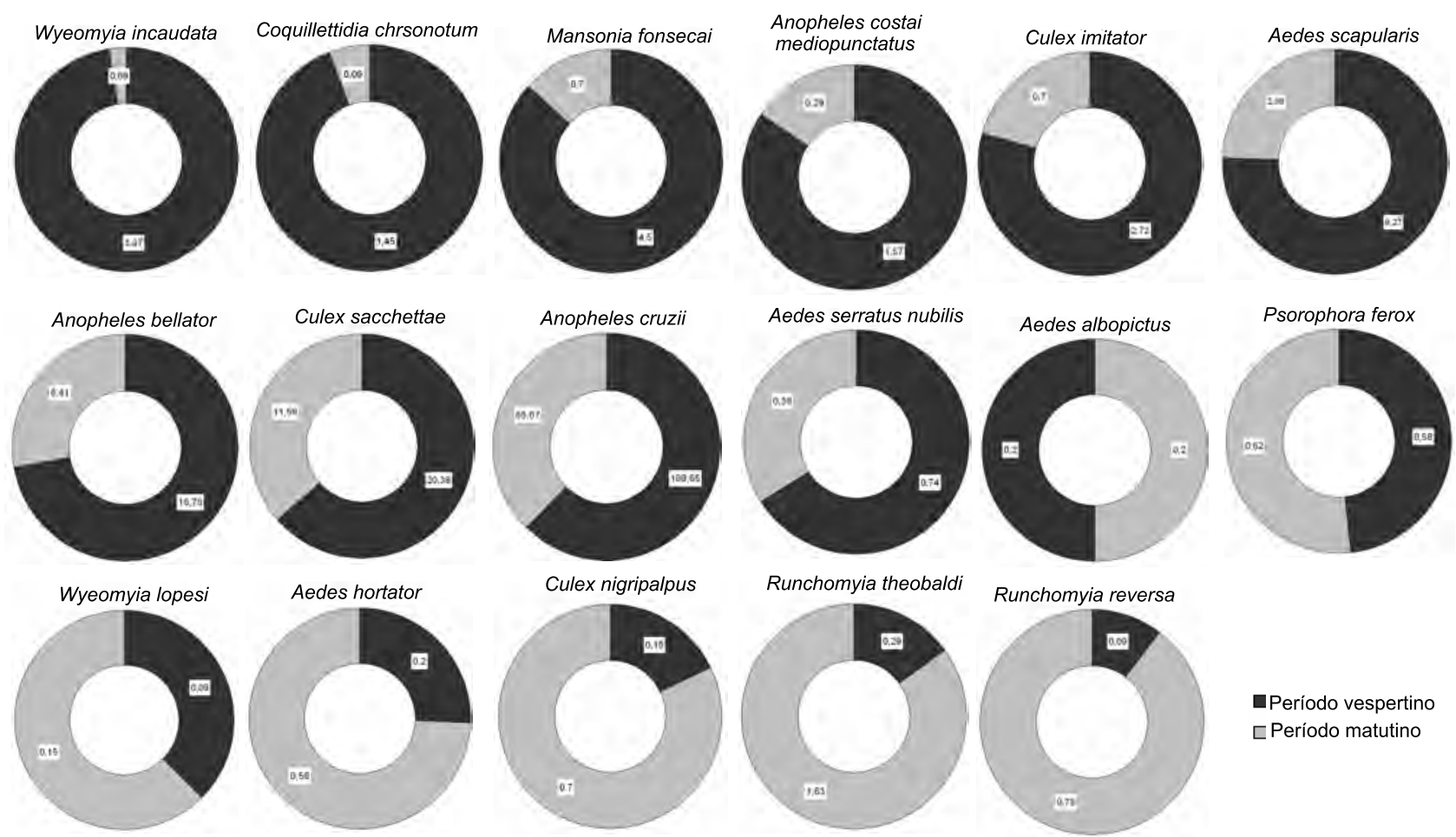

Figura 1. Freqüência das espécies de Culicidae, de acordo com a Média de Willians (Xw), no período crepuscular vespertino e matutino, entre dezembro de 2006 a março de 2007, na Floresta Estadual do Palmito, município de Paranaguá, Paraná.

Tabela II. Culicídeos capturados no período crepuscular vespertino e matutino, entre dezembro de 2006 a março de 2007, na Floresta Estadual do Palmito, Paranaguá, Paraná, com os respectivos percentuais de incidência, médias horárias (mH) e de Williams (Xw). Esforço de coleta de $20 \mathrm{~h}$.

\begin{tabular}{|c|c|c|c|c|c|c|c|c|}
\hline \multirow{3}{*}{ Espécies } & \multicolumn{8}{|c|}{ Período crepuscular } \\
\hline & \multicolumn{4}{|c|}{ Vespertino } & \multicolumn{4}{|c|}{ Matutino } \\
\hline & $\mathrm{N}$ & $\%$ & $\mathrm{mH}$ & $X w$ & $\mathrm{~N}$ & $\%$ & $\mathrm{mH}$ & $X w$ \\
\hline \multicolumn{9}{|l|}{ Anophelinae } \\
\hline Anopheles cruzii & 1193,00 & 60,04 & 59,65 & 108,65 & 1075,00 & 72,05 & 53,75 & 65,07 \\
\hline Anopheles bellator & 209,00 & 10,52 & 10,45 & 16,78 & 87,00 & 5,83 & 4,35 & 6,41 \\
\hline Anopheles mediopunctatus & 24,00 & 1,21 & 1,20 & 1,57 & 3,00 & 0,20 & 0,15 & 0,29 \\
\hline \multicolumn{9}{|l|}{ Culicinae: Aedini } \\
\hline Aedes scapularis & 110,00 & 5,55 & 5,50 & 9,23 & 32,00 & 2,14 & 1,60 & 2,98 \\
\hline Aedes serratus & 10,00 & 0,50 & 0,50 & 0,74 & 12,00 & 0,80 & 0,60 & 0,38 \\
\hline Psorophora ferox & 7,00 & 0,35 & 0,35 & 0,58 & 7,00 & 0,47 & 0,35 & 0,62 \\
\hline Aedes hortator & 2,00 & 0,10 & 0,10 & 0,20 & 6,00 & 0,40 & 0,30 & 0,58 \\
\hline Aedes albopictus & 2,00 & 0,10 & 0,10 & 0,20 & 2,00 & 0,13 & 0,10 & 0,20 \\
\hline Psorophora albipes & 1,00 & 0,05 & 0,05 & 0,09 & - & - & - & - \\
\hline Aedes fluviatilis & - & - & - & - & 1,00 & 0,07 & 0,05 & 0,09 \\
\hline \multicolumn{9}{|l|}{ Culicinae: Culicini } \\
\hline Culex sacchettae & 241,00 & 12,13 & 12,05 & 20,38 & 147,00 & 9,86 & 7,35 & 11,59 \\
\hline Culex imitator & 43,00 & 2,16 & 2,15 & 2,72 & 8,00 & 0,54 & 0,40 & 0,70 \\
\hline Culex grupo coronator & 3,00 & 0,15 & 0,15 & 0,20 & - & - & - & $\begin{array}{c}- \\
\text { Continua }\end{array}$ \\
\hline
\end{tabular}

Revista Brasileira de Zoologia 25 (1): 40-48, March, 2008 
Tabela II. Continuação.

\begin{tabular}{|c|c|c|c|c|c|c|c|c|}
\hline \multirow{3}{*}{ Espécies } & \multicolumn{8}{|c|}{ Período crepuscular } \\
\hline & \multicolumn{4}{|c|}{ Vespertino } & \multicolumn{4}{|c|}{ Matutino } \\
\hline & $\mathrm{N}$ & $\%$ & $\mathrm{mH}$ & Xw & $\mathrm{N}$ & $\%$ & $\mathrm{mH}$ & $X_{w}$ \\
\hline Culex ribeirensis & - & - & - & - & 3,00 & 0,20 & 0,15 & 0,26 \\
\hline Culex nigripalpus & 2,00 & 0,10 & 0,10 & 0,15 & 1,00 & 0,07 & 0,05 & 0,09 \\
\hline Culex quinquefasciatus & 1,00 & 0,05 & 0,05 & 0,09 & - & - & - & - \\
\hline \multicolumn{9}{|l|}{ Culicinae: Mansoniini } \\
\hline Mansonia fonsecai & 52,00 & 2,62 & 2,60 & 4,50 & 9,00 & 0,60 & 0,45 & 0,70 \\
\hline Coquillettidia chrysonotum & 16,00 & 0,80 & 0,80 & 1,45 & 1,00 & 0,07 & 0,05 & 0,09 \\
\hline \multicolumn{9}{|l|}{ Culicinae: Sabethini } \\
\hline Wyeomyia incaudata & 65,00 & 3,27 & 3,25 & 3,37 & 67,00 & 4,50 & 3,35 & 1,95 \\
\hline Runchomyia theobaldi & 4,00 & 0,20 & 0,20 & 0,29 & 16,00 & 1,07 & 0,80 & 1,63 \\
\hline Runchomyia reversa & 1,00 & 0,05 & 0,05 & 0,09 & 9,00 & 0,60 & 0,45 & 0,78 \\
\hline Wyeomyia lopesi & 1,00 & 0,05 & 0,05 & 0,09 & 2,00 & 0,13 & 0,10 & 0,15 \\
\hline Wyeomyia abebela & - & - & - & - & 2,00 & 0,13 & 0,10 & 0,20 \\
\hline Wyeomyia davisi & - & - & - & - & 1,00 & 0,07 & 0,05 & 0,09 \\
\hline Wyeomyia galvaoi & - & - & - & - & 1,00 & 0,07 & 0,05 & 0,09 \\
\hline Total & 1987,00 & 100,00 & 99,35 & 217,78 & 1492,00 & 100,00 & 74,60 & 124,89 \\
\hline
\end{tabular}
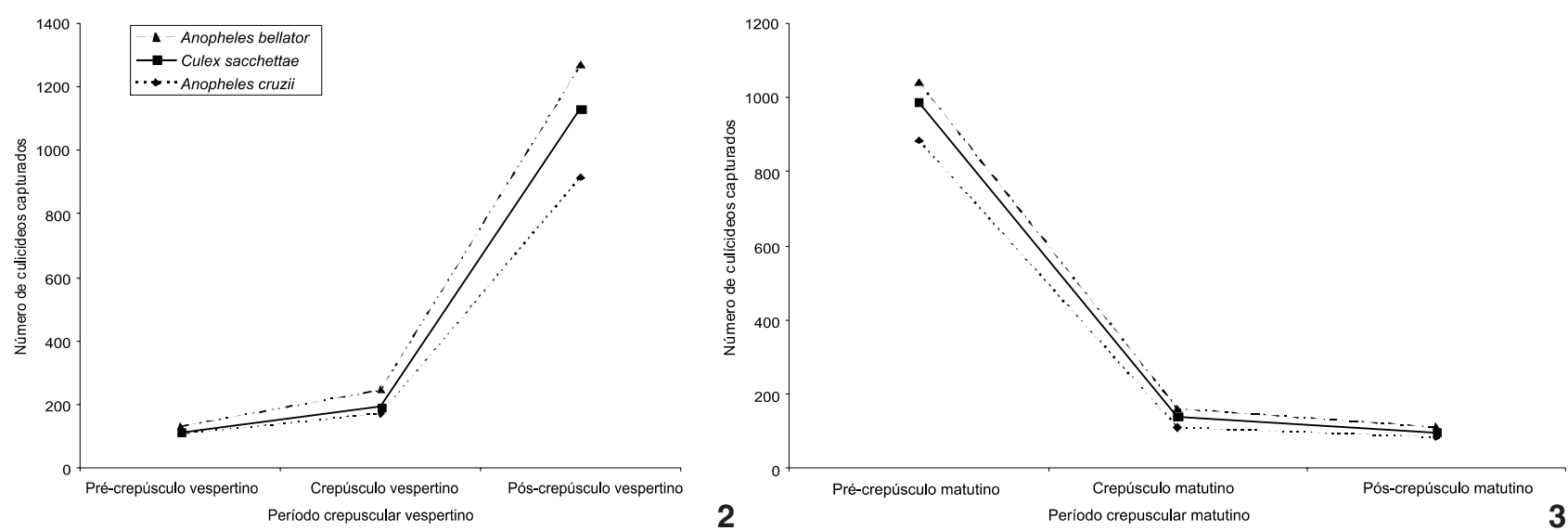

Figuras 2-3. Freqüência de Anopheles cruzii, A. bellator e Culex sacchettae capturados durante o período crepuscular vespertino (2) e matutino (3), entre dezembro de 2006 a março de 2007, na Floresta Estadual do Palmito, município de Paranaguá, Paraná.

clima variam de maneira sazonal, com temperatura média anual de $25^{\circ} \mathrm{C}$ e pluviosidade chegando a ultrapassar os $1000 \mathrm{~mm}$ (ForatTini 2002). De acordo com as médias históricas do Iapar a temperatura média entre 1978 e 1999 oscilou entre $16,2^{\circ} \mathrm{C}$ em julho a $24,5^{\circ} \mathrm{C}$ em fevereiro.

Para análise das correlações entre as variáveis ambientais e a freqüência de Culicidae, foram utilizadas as três espécies mais abundantes. Nenhuma apresentou valor de correlação de Pearson significativo. No entanto, os valores mais elevados obtidos de correlação positiva foram para Culex sacchettae e a média da temperatura média para 20 dias $(\mathrm{r}=0,69)$ e Culex sacchettae e a média da temperatura máxima para 20 dias $(\mathrm{r}=$ 0,72) (Tab. III).

A taxa de paridade em $A$. cruzii variou de 30,23 a 59,68 \% (Fig. 4), sendo negativa a correlação $(r=-0,23)$ entre a abundância e a taxa de paridade. A correlação entre a taxa de paridade e a média da umidade relativa foi negativa para cinco dias $(\mathrm{r}=-0,54)$. Para precipitação acumulada para cinco dias e a taxa de paridade a correlação foi positiva $(\mathrm{r}=-0,52)$, durante as oito coletas realizadas (Tab. IV). 


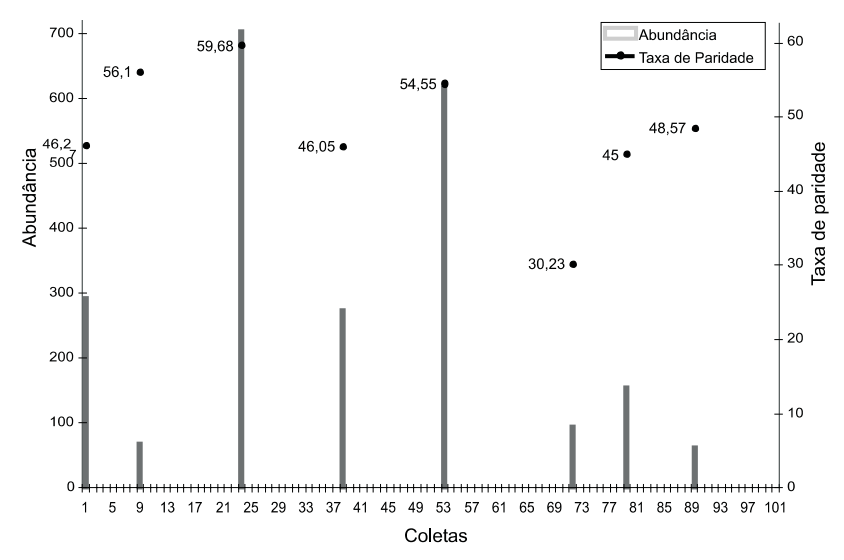

Figura 4. Freqüência de Anopheles cruzii e a taxa de paridade das fêmeas dissecadas, segundo a técnica de Detinova, entre dezembro de 2006 a março de 2007, na Floresta Estadual do Palmito, município de Paranaguá, Paraná.

Tabela III. Coeficiente de correlação de Pearson entre as médias das variáveis meteorológicas e as espécies mais abundantes, entre dezembro de 2006 a março de2007, na Floresta Estadual do Palmito, município de Paranaguá, Paraná. ( $T_{\text {méd' }} T_{\text {máx }}$ e $T_{\text {mín }}$ ) Temperatura média, máxima e mínima; $\left(U_{\text {rel }}\right)$ umidade relativa do ar; $\left(\right.$ Prec $\left._{\text {acum }}\right)$ precipitação pluviométrica acumulada, retrospectiva para 5,15 e 20 dias antes das coletas.

\begin{tabular}{|c|c|c|c|c|c|}
\hline & $\mathrm{T}_{\text {méd }}\left({ }^{\circ} \mathrm{C}\right)$ & $\mathrm{T}_{\text {máx }}\left({ }^{\circ} \mathrm{C}\right)$ & $\mathrm{T}_{\text {mín }}\left({ }^{\circ} \mathrm{C}\right)$ & $\mathrm{U}_{\mathrm{rel}}(\%)$ & $\operatorname{Prec}_{\text {acum }}(\mathrm{mm})$ \\
\hline \multicolumn{6}{|c|}{ Anopheles cruzii } \\
\hline 5 dias & $-0,34$ & $-0,43$ & $-0,66$ & 0,04 & 0,25 \\
\hline 15 dias & $-0,39$ & $-0,51$ & $-0,16$ & 0,30 & 0,10 \\
\hline 20 dias & $-0,28$ & $-0,40$ & 0,14 & 0,41 & 0,48 \\
\hline \multicolumn{6}{|c|}{ Anopheles bellator } \\
\hline 5 dias & $-0,40$ & $-0,53$ & $-0,47$ & 0,25 & 0,22 \\
\hline 15 dias & $-0,48$ & $-0,63$ & 0,10 & 0,59 & 0,15 \\
\hline 20 dias & $-0,34$ & $-0,45$ & 0,30 & 0,58 & 0,56 \\
\hline \multicolumn{6}{|c|}{ Culex sacchettae } \\
\hline 5 dias & 0,01 & 0,20 & 0,28 & 0,13 & 0,26 \\
\hline 15 dias & 0,63 & 0,61 & 0,62 & $-0,27$ & 0,24 \\
\hline 20 dias & 0,69 & 0,72 & 0,43 & $-0,57$ & 0,02 \\
\hline
\end{tabular}

Tabela IV. Coeficiente de correlação de Pearson entre as médias das variáveis abióticas, retrospectiva para 5, 15 e 20 dias antes das coletas, e a taxa de paridade de Anopheles cruzii, entre dezembro de 2006 a março de 2007, na Floresta Estadual do Palmito, Paranaguá, Paraná. ( $T_{\text {méd }}, T_{\text {máx }}$ e $T_{\text {mín }}$ ) Temperatura média, máxima e mínima; $\left(U_{\text {rel }}\right)$ umidade relativa do ar; $\left(\operatorname{Prec}_{\text {acum }}\right)$ precipitação pluviométrica acumulada.

\begin{tabular}{lccccc} 
Retrospectiva & $\mathrm{T}_{\text {méd }}\left({ }^{\circ} \mathrm{C}\right)$ & $\mathrm{T}_{\text {máx }}\left({ }^{\circ} \mathrm{C}\right)$ & $\mathrm{T}_{\text {mín }}\left({ }^{\circ} \mathrm{C}\right)$ & $\mathrm{U}_{\text {rel }}(\%)$ & $\operatorname{Prec}_{\text {acum }}(\mathrm{mm})$ \\
\hline 5 dias & $-0,50$ & 0,25 & $-0,47$ & $-0,54$ & 0,52 \\
15 dias & 0,28 & $-0,09$ & $-0,04$ & 0,39 & $-0,08$ \\
20 dias & 0,12 & $-0,07$ & $-0,04$ & 0,22 & 0,08 \\
\hline
\end{tabular}

\section{DISCUSSÃO}

Os dois períodos crepusculares apresentaram semelhança qualitativa e quantitativa. A porcentagem de similaridade foi de $85 \%$ e o índice de Morisita atingiu 0,98 sendo influenciados fortemente pela dominância de Anopheles cruzii. Para os culicídeos a queda e ascensão da luminosidade durante os crepúsculos vespertino e matutino respectivamente parecem ser fatores de desencadeamento das atividades (LourENÇO-DE-OLIVEIRA \& Silva 1985), para a maioria das espécies.

Os índices de dominância para o período crepuscular vespertino e matutino foram considerados elevados pela dominância de $A$. cruzii em relação as demais espécies nestes períodos. Quanto ao índice de diversidade de Margalef, os valores encontrados foram inferiores a cinco, denotando baixa diversidade de espécies nos dois períodos crepusculares, em conseqüência da reduzida freqüência da maioria das espécies capturadas.

As duas espécies de Anopheles capturadas em elevada densidade, $A$. cruzii e $A$. bellator, possuem em comum o desenvolvimento em criadouros naturais, preferencialmente na água acumulada nas imbricações das folhas das bromélias (Veloso et al. 1956). Existindo associação direta destas espécies com a oferta de criadouros, presentes na Floresta Estadual do Palmito. Quanto aos representantes de Anopheles, podem-se observar picos de atividade, tanto nos crepúsculos vespertinos como nos matutinos na área de Planície do Vale do Ribeira (Forattini et al. 1986). A influência crepuscular no desencadeamento da atividade culicídea foi reforçada nestas observações, com Culex sacchettae mostrando de maneira evidente o caráter eocrepuscular do seu ciclo nictimeral.

Na tribo Culicini, Culex sacchettae foi capturada em elevada densidade, no entanto, sua biologia ainda não é bem conhecida pouco se sabe sobre o criadouro preferencial desta espécie, sendo indicado coleção líquida com vegetação submersa, com distribuição indicada para regiões Sul e Sudeste. Alguns estudos em ambiente antrópico intensamente modificado indicaram elevado grau de sinantropia por parte desta espécie, sendo encontrada em apreciável freqüência em coletas que utilizam isca humana. (Forattini et al. 1991, Forattini 2002).

A atividade dos Aedini no período crepuscular vespertino foi mais elevada em comparação ao matutino, com destaque para Aedes scapularis, com forte presença para o vespertino. Lourenço-De-Oliveira \& Silva (1985), Forattini et al. (2000) e SANT'ANA \& Lozovei (2001) consideram esta espécie eclética, com aumento da densidade no final do crepúsculo vespertino. Forattini et al. (1986) consideram Aedes serratus e Psorophora ferox (Von Humbold, 1819) essencialmente diurnas. No entanto, observamos estas espécies em baixa freqüência e com a mesma densidade nos períodos vespertino e matutino, sugerindo o término e ínicio de suas atividades.

A presença significativa de Aedes scapularis, espécie adaptável a ambientes modificados (ForatTini et al. 1986), possibilita a interpretação de que a preservação da área ainda não atin- 
giu o clímax além de sofrer influência de atividade antrópica principalmente no seu entorno.

Os Mansoniini foram encontrados em maior densidade no período crepuscular vespertino. ForatTinI (2002) relata que a tribo tem picos de atividades crepusculares, principalmente no período vespertino. Coquillettidia chrysonotum tem preferência crepuscular vespertina como observado por GUIMARÃEs et al. (2000).

Os Sabethini tiveram predominância no período crepuscular matutino, principalmente Runchomyia theobaldi que tem hábito predominantemente diurno como relatado em PATERNo \& Marcondes (2004). As espécies de Wyemyia também merecem destaque, são de atividade diurna, mas estendem sua atividade até o início da noite (Foratrini et al. 1968).

As espécies coletadas em abundância elevada como A. cruzii e $A$. bellator, representantes dos Anophelinae, tiveram um aumento gradativo de densidade com a redução ou aumento da intensidade da luz, sendo este um estímulo exógeno e a isca humana um estímulo endógeno. Segundo Aragão (1974) a atividade dos Anopheles é intensificada no pôr do sol. A variação bimodal crepuscular, alternância dos picos de atividade no amanhecer e no anoitecer (SAN't ANA \& Lozover 2001), parece ter ocorrido com $A$. cruzii e $A$. bellator, já que foram encontradas em elevada abundância no pós-crepúsculo vespertino e pré-crepúsculo matutino.

Os horários crepusculares funcionam como o momento de mudança do comportamento das espécies de mosquitos encerrando ou iniciando suas atividades. As fêmeas de Wyeomyia incaudata foram encontradas em densidade considerável durante os períodos crepusculares, apontando para comportamento diurno, como já relatado em (Marcondes \& Paterno 2005), iniciando suas atividades no crepúsculo matutino e encerrando no crepúsculo vespertino. Diferentemente dos Anpheles e Culex sacchettae, que parecem iniciar suas atividades com a chegada do anoitecer e diminuem suas atividades no amanhecer.

As variáveis ambientais influenciam fortemente a atividade de culicídeos. Na estação do verão nas regiões tropicais as variáveis abióticas geralmente são favoráveis aos Culicidae, pois com a ocorrência de chuvas, a oferta de criadouros é aumenta$\mathrm{da}$, e as temperaturas elevadas facilitam o ciclo de desenvolvimento dos insetos. GuIMARÃEs et al. (2000) relatam que as variações da temperatura e umidade relativa do ar influenciam diretamente os mosquitos e estes podem chegar a desaparecer totalmente durante os meses mais secos do ano.

Não encontramos correlações significativas entre a abundância das espécies e a média dos fatores abióticos, durante o período de investigação os fatores climáticos apresentaram comportamento praticamente homogêneo. As médias de temperatura média, máxima, mínima e a umidade relativa do ar sofreram reduzida oscilação. E a precipitação acumulada foi elevada no decorrer do período analisado.

Dentre as fêmeas de $A$. cruzii dissecadas, $48 \%$ já haviam realizado pelo menos um repasto sanguíneo. JENSEN et al. (1998) consideram que elevada taxa de paridade indica maior longevidade da população. DiéGuEz et al. (2002) sugerem que a abun- dância pode ser um reflexo da idade fisiológica das fêmeas. No entanto, de acordo com as observações efetuadas não ocorreu correlação entre a taxa de paridade e a abundância de $A$. cruzii. A taxa de paridade está correlacionada positivamente com a precipitação acumulada para cinco dias precedentes à coleta, pois com o aumento das chuvas, ocorre o abastecimento dos criadouros, tornando-os propícios para o desenvolvimento dos imaturos desta espécie. Este cenário pode desencadear o comportamento de busca de hospedeiro para o exercício da hematofagia.

As espécies encontradas buscam exercer hematofagia sobre hospedeiro humano, podendo atuar com vetoras de agentes patogênicos. Algumas já possuem informações sobre aspectos de seu comportamento, biologia e epidemiologia, porém outras ainda necessitam de investigação. A intensificação de estudos abordando a ecologia desta família de Diptera torna-se necessária diante da intensidade das transformações ambientais provocadas pelo homem, que podem determinar a criação de novos cenários epidemiológicos.

O período crepuscular exerce influência determinante no comportamento apetente de muitas espécies de Culicidae, indicando o início ou final da atividade determinando o período de atividade preferencial diurno ou noturno. A comparação de similaridade entre os crepúsculos apontou para elevada semelhança da composição específica. Anopheles cruzii atingiu dominância nos períodos crepusculares, sendo a diversidade de forma geral reduzida nestes períodos.

\section{AGRADECIMENTOS}

Ao Sistema Meteorologia do Paraná (Simepar) pelo fornecimento dos dados climáticos. À Maria A.M. Sallum, Faculdade de Saúde Pública (USP) pela conferência da identificação das espécies. Ao CNPq.

\section{LITERATURA CITADA}

Aragão, M.B. 1974. O comportamento dos anofelinos do subgênero Kerteszia, no Sul do Brasil e o efeito do inseticida DDT. Memórias Instituto Oswaldo Cruz 72: 147-172.

Barbosa, A.A.; M.A.N. Silva; M.A.N. Navarro-Silva \& M.A.M. SAllum. 2005. Description and revalidation of Mansonia (Mansonia) fonsecai (Pinto) (Diptera: Culicidae). Zootaxa 905: 1-11.

Berger W.H. \& F.L. Parker. 1970. Diversity of plantonic Foraminifera in deep sea sediments. Science 168: 1345-1347.

Boeger, M.R.T. \& C. Winiewiski. 2002. Estrutura e teores de nutrientes foliares de seis espécies arbóreas ao longo de um gradiente sucessional da planície litorânea do Estado do Paraná. Ilheringia 57: 243-262.

Boeger, M.R.T. \& C. Winiewiski. 2003. Comparison of leaf morphology of tree species from three distinct sucessional stages of tropical rain forest (Atlantic Forest) in Southern Brazil. Revista Brasileira de Botânica 1: 61-72.

Carvalho-Pinto, C.J. \& R. Lourenço de Oliveira. 2004. Isoenzimatic Analysis of four Anopheles (Kerteszia) cruzii populations of 
Brazil. Memórias Instituto Oswaldo Cruz 99 (5): 471-475.

Chahad-Ehlers, S.; A.L. Lozovei \& M.D. Marques. 2007. Reproductive and post-embrionic daily rhythm patterns of the malaria vector Anopheles (Kerteszia) cruzii: Aspects of the lyfe cycle. Chronobiology International 24: 289-304.

Charlwood, J.D.; J.A. Rafael \& T.J.Wilkes. 1980. Métodos de determinar a idade fisiológica em Diptera de importância médica. Uma revisão com especial referência aos vetores de doenças na América do Sul. Acta Amazonica 10: 311-333.

Consoli, R.A. \& R. Lourenço-de-Oliveira. 1994. Principais espécies de importância sanitária no Brasil. Rio de Janeiro, Fiocruz, 225p.

CorrêA, R.R. \& G.R. Ramalho. 1956. Revisão de Phoniomyia Theobaldi, 1903 (Diptera, Culicidae, Sabethini). Fiolia Clinica et Biológica 25: 1-176.

Detinova, T.S. 1962. Age-grading methods in Diptera of medical importance. Geneva, WHO, Monographies Series 47, 216p.

Dieguez, L.; R. Rodriguez; E. Atienzar; O. Manso; P. Basulto \& N. GóNGORA. 2002. Observaciones entomológicas en un brote de paludismo durante la etapa de vigilancia intensiva en Albaisa, Camagüey. Revista Cubana Medicina Tropical 54: 118-126.

Felfili, J.M.; M.C. Silva-Júnior; A.V. Rezende; J.W.B. Machado; B.M.T. Walter; P.E.N. Silva \& J.D. Hay. 1993. Análise comparativa da florística e fitossociologia da vegetação arbórea do cerrado sensu stricto na Chapada Pratinha, DF-Brasil. Acta Botânica Brasileira 6: 27-47.

Forattini, O.P. 2002. Culicidologia Médica. São Paulo, Editora da Universidade de São Paulo, vol. 2, 860p.

Forattini, O.P.; O.S. Lopes \& E.X. Rabello. 1968. Investigações sobre o comportamento de formas adultas de mosquitos silvestres no Estado de São Paulo, Brasil. Revista de Saúde Pública 2 (2): 111-173.

Forattini, O.P.; A.C. Alves; D. Natal \& J.L.F. Santos. 1986. Observações sobre atividade de mosquitos Culicidae em mata primitiva da encosta no Vale do Ribeira, São Paulo, Brasil. Revista de Saúde Pública 20 (1): 1-20.

Forattini, O.P.; A.C. Gomes; I. KaKitani \& D. Marucci. 1991. Observações sobre a domiciliação de mosquitos Culex (Melanonoconion), em ambiente com acentuadas modificações antrópicas. Revista de Saúde Pública 25: 257-266.

Forattini, O.P.; I. KaKitani; R.L.C. Santos; K.M. Kobayashi; H.M. Ueno \& Z. Fernandez. 2000. Comportamento de Aedes albopictus e de Ae. scapularis adultos (Diptera: Culicidae) no Sudeste do Brasil. Revista de Saúde Pública 34 (5): 461-467.

Guimarães, A.E.; C. Gentile; C.M. Lopes \& R.P. Mello. 2000. Ecology of mosquitoes (Diptera: Culicidae) in areas of Serra do Mar State Park, State of São Paulo, Brazil. III - daily biting rhythms and lunar cycle influence. Memórias Instituto Oswaldo Cruz 95 (6): 753-760.

Hoeck, P.A.E.; F.B. Ramberg; S.A. Merrill; C. Moll \& H.H. Hagedorn. 2003. Population and pariry levels of Aedes aegypti collect in Tucson. Journal of vector Ecology 28 (1): 1-9.

Hutchings, R.S.G.; M.A.M. Sallum; R.L.M. Ferreira \& R.W.
Hutchings. 2005. Mosquitoes of the Jaú Park and their potential importance in Brazilian Amazonia. Medical and Veterinary Entomology 19: 428-441.

IAPAR. Cartas climáticas do Paraná. 2002. Disponível em: http:/ /200.201.27.14/Site/Sma/Cartas_Climaticas/Classificacao_ Climatica.htm [Acessado em: 10/III/2008]

Jensen, T.; D.A. Dritz; G.N. Fritz; R.K. Washino \& W.C. Reeves. 1998. Lake Vera revisited: parity and survival rates of Anopheles punctipennis at the site of a malaria outbreak in the Sierra Nevada foothills of California. American Journal Tropical Medicine and Hygiene 59: 591-594.

LANE, J. 1953a. Neotropical Culicidae. Sào Paulo, Editora da Universidade de São Paulo, vol. 1, 548p.

LANE, J. 1953b. Neotropical Culicidae. São Paulo, Editora da Universidade de São Paulo,vol. 2, 564p.

Lane, J. \& L. Whitman. 1951. The subgenus "Microculex" in Brazil (Diptera: Culicidae). Revista Brasileira de Biologia 11: 341-366.

LourenÇO-De-Oliveira, R. \& T.F. Silva. 1985. Alguns aspectos da ecologia dos mosquitos (Diptera: Culicidae) de uma área de planície (Granjas Calábria), em Jacarepaguá, Rio de Janeiro. Preferência horária das fêmeas para o hematofagismo. Memórias Instituto Oswaldo Cruz 80: 195-201.

Marcondes, C.B. \& U.PAterno. 2005. Preliminary evidence of association between species of mosuitoes in Atlantic Forest of Santa Catarina State, (Diptera: Culicidae). Revista da Sociedade Brasileira de Medicina Tropical 38: 75-76.

Paterno, U. \& C.B. Marcondes. 2004. Mosquitoes with morning biting activity in the Atlantic forest, Brazil (Diptera, Culicidae). Revista de Saúde Pública 38 (1): 133-135.

RAchou, R.G. 1958. Anofelinos do Brasil: comportamento das espécies vetoras de malária. Revista Brasileira de Malariologia e Doenças Tropicais 10:145-181.

Roderjan, C.V.; F. Galvão; Y.S. KuniYoshi \& G.G. Hatcshbach. 2002. As unidades fitogeográficas do estado do Paraná. Ciência e Ambiente 24: 75-92.

SAnT'ANA, A.L. \& A.L. Lozover. 2001. Influência do ciclo lunar na captura de Aedes scapularis (Diptera: Culicidae) na Mata Atlântica do Paraná. Ilheringia 90: 175-182.

Service, M.W. 1993. Mosquito ecology: field sampling methods. London, Chapman Hall, 988p.

Simões, C.G. \& M.C.M. Marques. 2007. The role sprouts in the restoration of Atlantic Rainforest in Southern Brazil. Restoration Ecology 15: 53-59.

Veloso, H.P.; J.V. Moura \& R.M. Klein. 1956. Delimitação ecológica dos anofelíneos do subgênero Kerteszia na região costeira do sul do Brasil. Memórias Instituto Oswaldo Cruz 54: 517-549.

Volpato, G.H. \& L. dos Anjos. 2001. Análises das estratégias de forrageamento das aves que se alimentam no solo na universidade estadual de Londrina, Estado do Paraná. Ararajuba 9: 95-99.

Received in 16.X.2007; accepted in 10.III.2008. 